\title{
Role of humidity in reducing the friction of graphene layers on textured surfaces
}

\author{
Zheng-yang $\mathrm{Li}^{\mathrm{a}}$, Wen-jing Yang ${ }^{\mathrm{a}}$, Yan-ping $\mathrm{Wu}^{\mathrm{b}}{ }^{\mathrm{w}}$, Song-bo $\mathrm{Wu}^{\mathrm{a}}$, Zhen-bing Cai ${ }^{\mathrm{a}, *}$ \\ ${ }^{\mathrm{a}}$ Tribology Research Institute, Key Lab of Advanced Technologies of Materials, \\ Southwest Jiaotong University, Chengdu, 610031, China \\ ${ }^{\mathrm{b}}$ China Academy of Engineering and Physics, Mianyang, 621900, China \\ *Corresponding authors. E-mail addresses: caizb@swjtu.cn (Z.-b. Cai), \\ wuyanping-2@126.com (Y.-p.Wu)
}

\begin{abstract}
A multiple-layer graphene was prepared on steel surface to reduce friction and wear. A graphene-containing ethanol solution was dripped on the steel surface, and several layers of graphene flakes were deposited on the surface after ethanol evaporated. Tribological performance of graphene-contained surface (GCS) was induced by reciprocating ball against plate contact in different RH ( $0 \%$ (dry nitrogen), $30 \%, 60 \%$, and 90\%). Morphology and wear scar were analyzed by OM, 2D profile, SEM, Raman spectroscopy, and XPS. Results show that GCS can substantially reduce the wear and coefficient of friction (COF) in $60 \%$ relative humidity $(\mathrm{RH})$. Low COF occurs due to graphene layer providing a small shear stress on the friction interface. Meanwhile, conditions of high RH and textured surface could make the low COF persist for a longer time. High moisture content can stabilize and protect the graphene C-network from damage due to water dissociative chemisorption with carbon dangling bonds, and the textured surface was attributed to release graphene layer stored in the dimple.
\end{abstract}

Key words: graphene; friction and wear; surface textured; humidity

\section{Introduction}


Since the early forties, it is well known that the lubricating property of graphite is derived from its laminar structure where carbon atoms lying on the same layer are closely packed and it is strongly environment dependent. Many experimental studies investigating the influence of the gas atmosphere on the friction regime of graphite [1-4], and graphite in water can lead to ultralow friction by three-step friction test [5]. Rietsch have indicated that graphite can afford a lower friction in moisture environments due to the presence of an oriented graphite friction layer which can be formed only if the carbon dangling bonds are deactivated by water dissociative chemisorption [6]. Highly oriented pyrolytic graphite (HOPG) similar to single crystalline graphite also Show a lower friction in the humid environments, and the COF of the perpendicular surface is higher than that of the parallel surface [7].

Graphene as a fascinating 2D interfacial material had attracted considerable attention from scientists because of its unique physical, electronic, and chemical properties [8-10].In theory, small shear force between the produced layers results in lower COF than that of graphite, thus possibly contributing to antifriction and lubrication [11]. Some authors investigate the micro-tribological behavior of graphene grown on $\mathrm{SiC}$ under different condition by a combination of a microtribometer with an AFM [12-15]. In the aspect of macro-scale, graphene coating deposited on the substrate is to reduce friction [16-17], and the COF of multilayered graphene is studied under different RH [18]. Berman et al. [19-22] conduct the tribological tests of graphene flakes deposited on steel surface sliding against steel balls at a normal load of $2.0 \mathrm{~N}$ and recorded a low COF of 0.15. Results show that this low COF can persist for 1200 cycles in $\mathrm{N}_{2}$ atmosphere and for 47000 cycles in $\mathrm{H}_{2}$ atmosphere because the hydrogen atoms can protect the underlying graphene surface from further damage. However, the report on the friction of graphene layer under different $\mathrm{RH}$ condition is fairly limited, especially on macroscale friction. The role and mechanism of water dissociative chemisorption with graphene layer during friction was not clearly. 
In the present research, a graphene-containing ethanol solution was dripped on the steel surface, and the steel surface formed several-layer graphene flakes after the liquid ethanol evaporated. The motivation of this report is to investigate the durability and degradation mechanism of GCS in different RH sliding condition and analyze the role and chemisorption mechanism of water during friction, which was not reported in previous works.

\section{Experimental procedure}

The single-layer graphene particles used in this research were purchased from Jinan Graphene New Materials. Graphene particles were added to the ethanol solution at a weight concentration of $1 \mathrm{mg} / \mathrm{L}$. Then, graphene + ethanol solution was stirred for over 10 min and subjected to 60 min of ultrasonic vibration for uniform suspension in the ethanol solution. A $0.15 \mathrm{~mL}$ solution of per $1 \mathrm{~cm}^{2}$ was applied on the steel surface in a droplet state, and the liquid ethanol part was evaporated in dry nitrogen environment to protect graphene from oxidation. Then, the steel surface would form multiple-layer graphene flakes (Fig. 1a), and the character of the graphene layer was observed by scanning electron microscopy (SEM) (JSM-7001F; JEOL, Japan). As shown in Fig. 1b, several graphene layers were irregularly distributed in the steel surface. The characteristic of graphene layer on the surface was analyzed by Raman spectrum (Lab Ram HR) characterization, utilizing a $532 \mathrm{~nm}$ laser as excitation source. Fig. 1c shows the Raman spectrum of the graphene layer deposited on the steel surface. Three peaks are clearly observed in Fig. 1c, particularly the D, G, and 2D peaks, which are the typical features of graphene. The D peak at $1320 \mathrm{~cm}^{-1}$ was known as the disorder band, which corresponds to the $s p^{2}$ hybridized health defects (graphene edge structure). The $\mathrm{G}$ peak at $1580 \mathrm{~cm}^{-1}$ indicates the vibration in all of the $s p^{2}$-bonded carbon atoms, thereby denoting a "defect-free" graphitic character. By contrast, the $2 \mathrm{D}$ peak at $2700 \mathrm{~cm}^{-1}$ reflects the thickness of graphene, especially for multiple-layer graphene. The ratio of $2 D / G$ intensity in Fig. 1c is below 1, indicating that approximately two- to four-layer graphene was deposited on the steel surface [23]. 
The presence of D peak is due to the partial oxidation of graphene during deposition as well as non-planar deposition of the flakes.

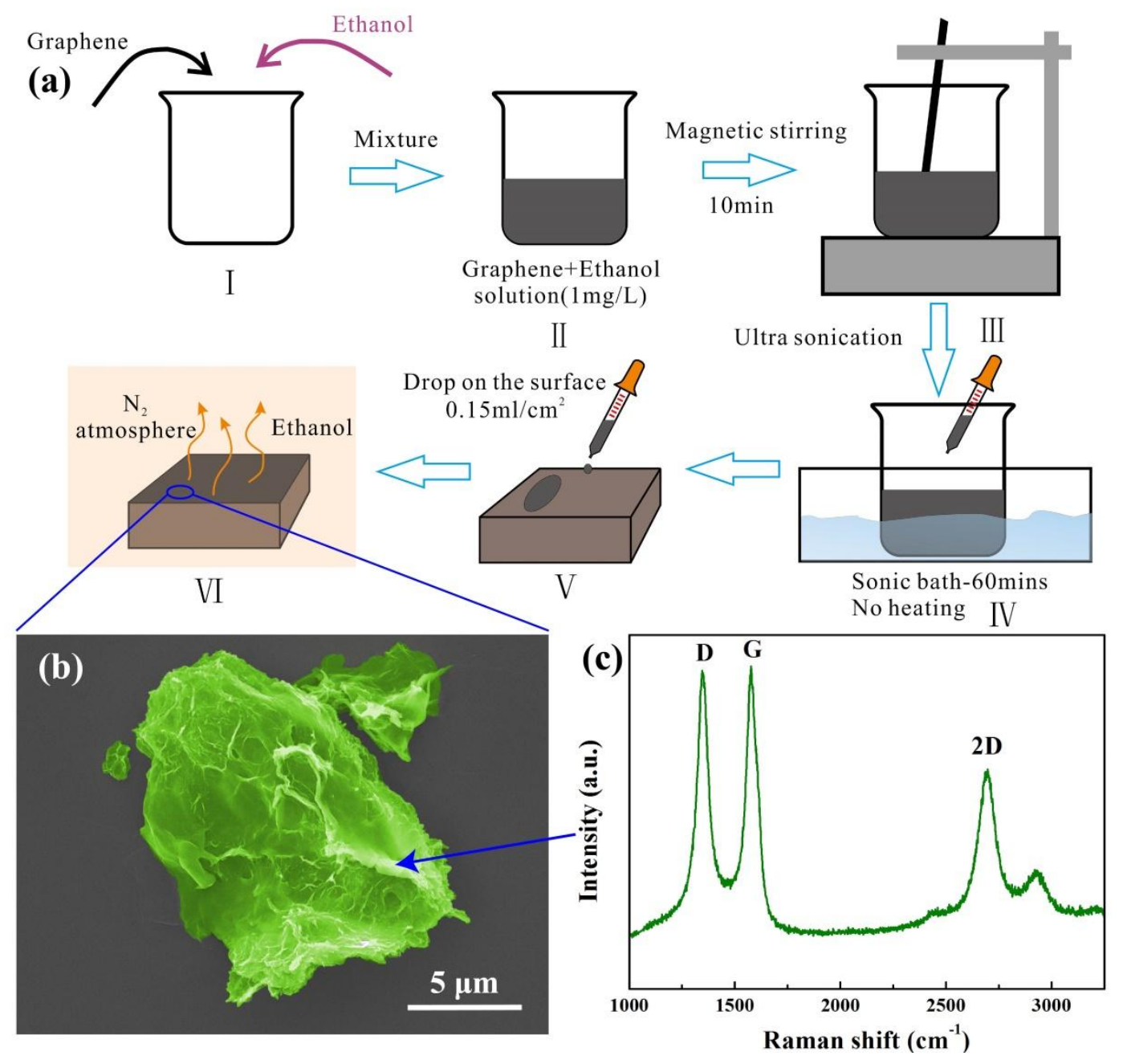

Fig. 1 Preparation process (a), SEM image (b), and Raman spectra (c) of GCS

The steel surface with $20 \%$ area ratio round dimples was prepared by laser etching with wavelength of $1064 \mathrm{~nm}$, average power of $10 \mathrm{~W}$, pulse frequency of 10 $\mathrm{kHz}$, and scan speed of $5 \mathrm{~mm} / \mathrm{s}$. When the laser beam hits the steel surface, a temperature gradient was generated on the surface. This temperature gradient induced the formation of a surface tension gradient toward the molten pool, causing the material to amass at the pool edge [24]. Fig. 2 shows the morphology and 2D profile of textured surface, and the dimples were $100 \mu \mathrm{m}$ in diameter and $20 \mu \mathrm{m}$ in depth. 


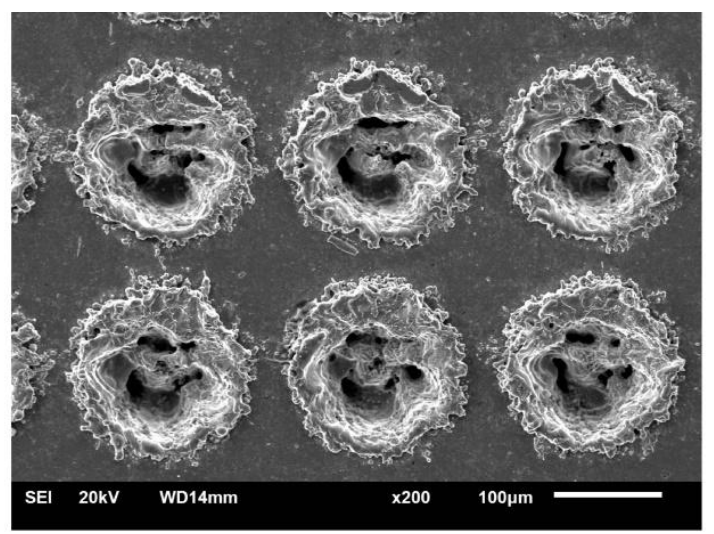

(a)

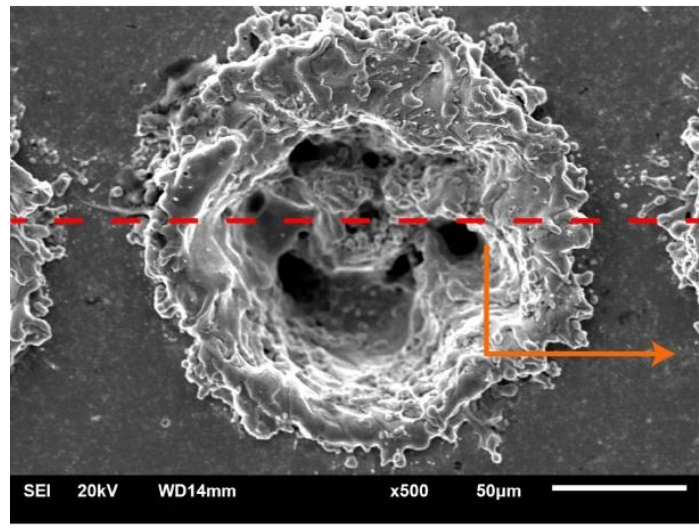

(b)

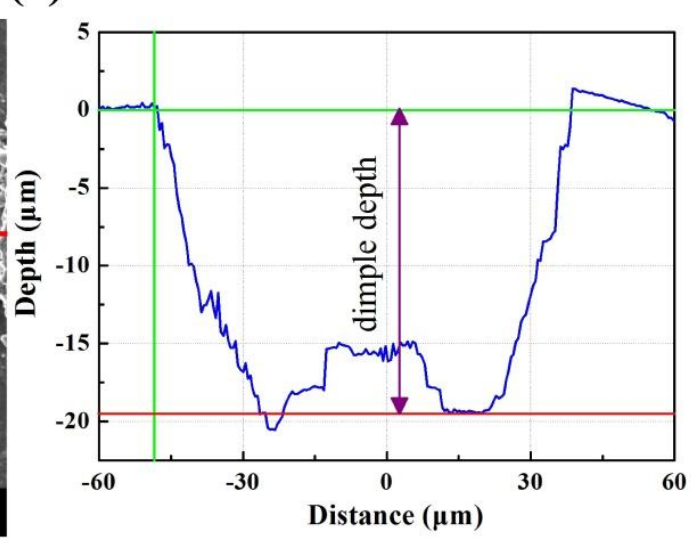

(c)

Fig. 2 Morphologies and 2D profile of the textured surface (a), dimple (b), and 2D profile (c).

Tribological performance was conducted with a ball-plate contact wear model by using a UMT-2 testing machine. The upper ball sample was GCr15 steel (wt.\%: $\mathrm{C}-1.0, \mathrm{Cr}-1.49, \mathrm{Mn}-0.31, \mathrm{Si}-0.26, \mathrm{P}-0.009$, and $\mathrm{S}-0.004)$ with a diameter of 9.525 mm and hardness of $766 \mathrm{HV}$. The lower plate specimen was $65 \mathrm{Mn}$ spring steel (wt.\%, $\mathrm{C}-0.68, \mathrm{Si}-0.25, \mathrm{Mn}-1.1, \mathrm{~S} \leqslant 0.035, \mathrm{P} \leqslant 0.035, \mathrm{Cr} \leqslant 0.2, \mathrm{Ni} \leqslant 0.25$, and $\mathrm{Cu}$ $\leqslant 0.25)$ with hardness of $270 \mathrm{HV}$. The roughness of the ball and plate was 0.4 and $1.5 \mu \mathrm{m}$, respectively. During the test, the normal load was $2 \mathrm{~N}$ (average Hertz contact pressure was approximately $0.45 \mathrm{GPa}$ ); the sliding speed was $8 \mathrm{~mm} / \mathrm{s}$; the reciprocating sliding distance was $8 \mathrm{~mm}$; and the test time was $1200 \mathrm{~s}$ in controlled RH atmosphere (0\% (dry nitrogen), 30\%, 60\%, and 90\%); The moisture content was monitored using a hygrometer with sensitivity of $\pm 0.1 \%$ of the desired $\mathrm{RH}$ level. The 
moisture content in the chamber was controlled by adding the proportion of water vapor and dry nitrogen. Each test was repeated for three times.

After the test, specimens were cleaned with acetone and ethanol, and then dried. The morphologies and tribochemistry of wear scars were analyzed through optical microscopy (OLYMPUS BX50), SEM (JSM-7001F; JEOL, Japan), energy dispersive X-ray spectroscopy (EDX, EDAX-7760/68M), Raman spectrum (Lab Ram HR) characterization, and X-ray photoelectron spectrum (XPS, thermo-250Xi). The wear volume and 2D profile were measured by nanomap (Aep), and wear rate was calculated.

\section{Results and discussion}

\subsection{Friction behavior of GCS}

The COF of bare surface and GCS under $60 \%$ RH condition are shown in Fig. 3a. The COF of bare surface rapidly increase to 0.6 and remain stable after the removal of oxides and the pollution layer of steel surface. In contrast to the bare surface, the COF of GCS was first decrease to a relatively low value of 0.16 and remains stable until 625s. The low COF indicates the lubrication effect of the graphene layer, and then increased to 0.55 is attributed to the gradual destruction of the graphene layer and graphene layer removal of wear scar during reciprocating sliding. However, the stabilized COF of GCS is lower than that of the bare surface because of the remaining amorphous carbon. These results are in accordance with the previous study reported by another group [19].

The optical microscopy images of wear scars are displayed in Fig. 3b- 3e. After 1200 cycles, the wear scar diameters of ball against the bare surface and plate are 425 and $338 \mu \mathrm{m}$, relatively. However, the wear scar diameter of the ball against GCS is lower only $292.5 \mu \mathrm{m}$, and the plate is $237 \mu \mathrm{m}$. Therefore, graphene layers can evidently 
reduce the friction and wear of the steel surface. Meanwhile, the diameter of the ball is always larger than the wear width of plate samples because the ball stays in contact all the time, whereas the plates are in contact periodically. From Fig. 3, the bare surface contains more black wear debris than that of GCS, and the wear debris in the wear scar is not removed, leading to bare surface presenting spikes of ups and downs according to the 2D profile. However, the GCS exhibit a slight damage, and the maximum wear depth is $0.4 \mu \mathrm{m}$ less than the bare surface of $1.5 \mu \mathrm{m}$.

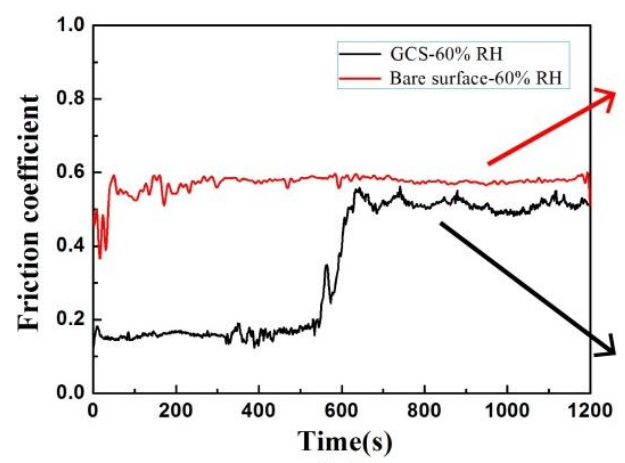

(a)

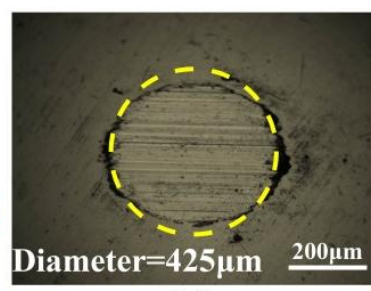

(b)

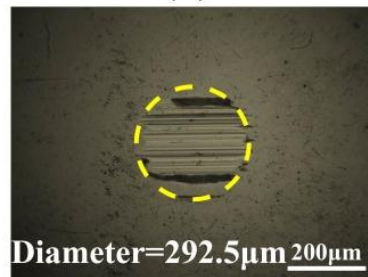

(d)

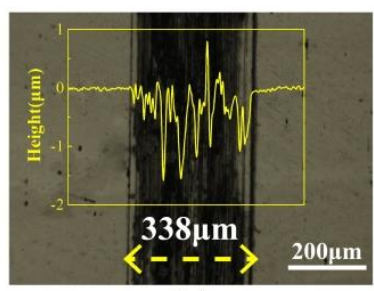

(c)

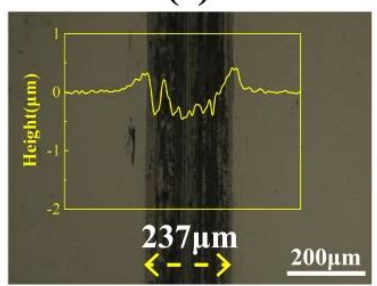

(e)

Fig. 3 COF of bare surface and GCS (a); the ball with bare surface plate (b and c) and ball with GCS (d and e) after $1200 \mathrm{~s}$ test in $60 \% \mathrm{RH}$.

\subsection{Friction behavior of textured GCS}

Fig. 4 shows the COF of the bare and textured surfaces, GCS, and textured GCS in different RH atmospheres. From Fig. 4b, the change of COF is basically the same in different $\mathrm{RH}$ atmospheres, which firstly decrease to 0.16 because of the lubrication of graphene flakes, then increase to a certain value, and remaining stable. The difference occurs at the duration of low COF under different test conditions only 265 $\mathrm{s}$ in dry $\mathrm{N}_{2}$ atmosphere and 440,625, and $871 \mathrm{~s}$ in 30\%,60\%, and 90\% $\mathrm{RH}$ atmosphere, respectively. The COF under $\mathrm{N}_{2}$ atmosphere fluctuates substantially due 
to severe adhesive wear and third-body wear debris in the friction interface. The COF of textured sample is shown in Fig. $4 \mathrm{c}$ and d, the textured sample presents a lower, steady, and smooth COF curve than that of the bare surface under all test conditions because the dimple in the surface can collect wear debris and reduce the third-body wear. Moreover, by contrast with GCS, the low COF of textured GCS lasts for a longer time under all test conditions, especially for $90 \% \mathrm{RH}$, which donot increase until 1200s. This phenomenon indicates that graphene layer on the textured surface was able to provide more long-term lubrication.

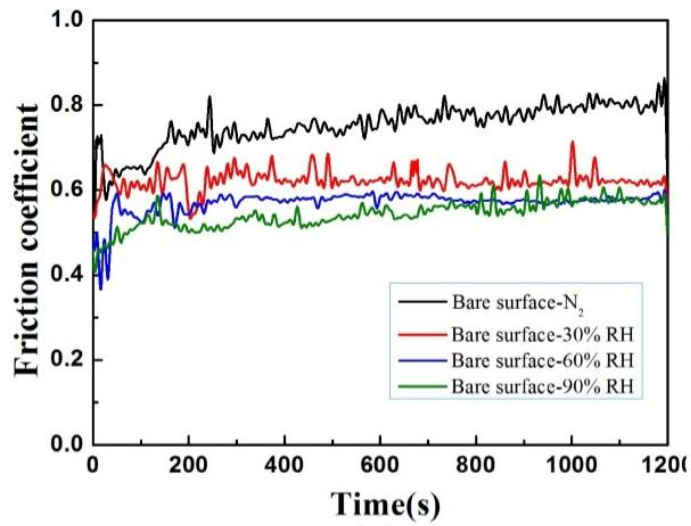

(a)

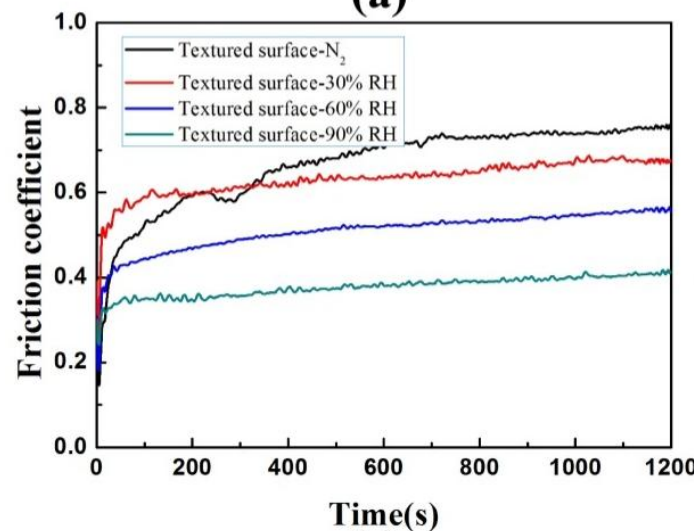

(c)

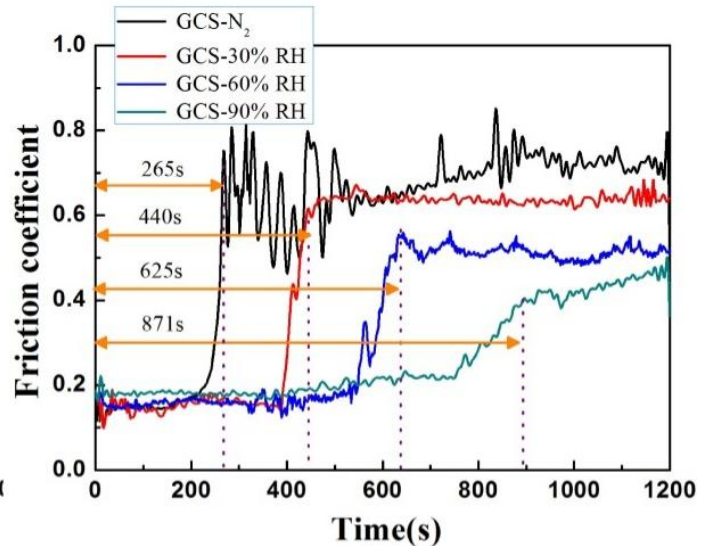

(b)

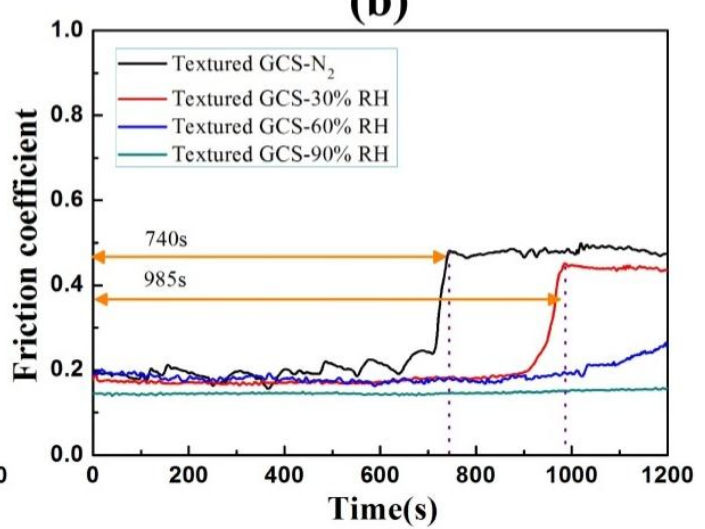

(d)

Fig. 4 COF of bare surface (a), GCS (b), textured surface (c), and textured GCS (d) under different $\mathrm{RH}$ condition

The average COF is shown in Fig. 5a. The average COF under all test conditions decreased with the increase in $\mathrm{RH}$ because of water lubrication, which protects the surface from friction. Meanwhile, the COF of GCS is always lower than that bare 
surface. The tendency of wear rate is listed in Fig. $5 \mathrm{~b}$ corresponding to the average COF. From Fig. 5b, the wear rate of GCS is below that of the bare surface under all test conditions. Moreover, the wear rate falls most considerably (about six times) at $90 \%$ $\mathrm{RH}$, decreasing from $37.08 \times 10^{-6} \mathrm{~mm}^{3} / \mathrm{Nm}$ to $6.25 \times 10^{-6} \mathrm{~mm}^{3} / \mathrm{Nm}$. However, the wear rate of the textured sample was difficult to measure owing to the existence of micro-dimples and slight damage, thus it is not listed.

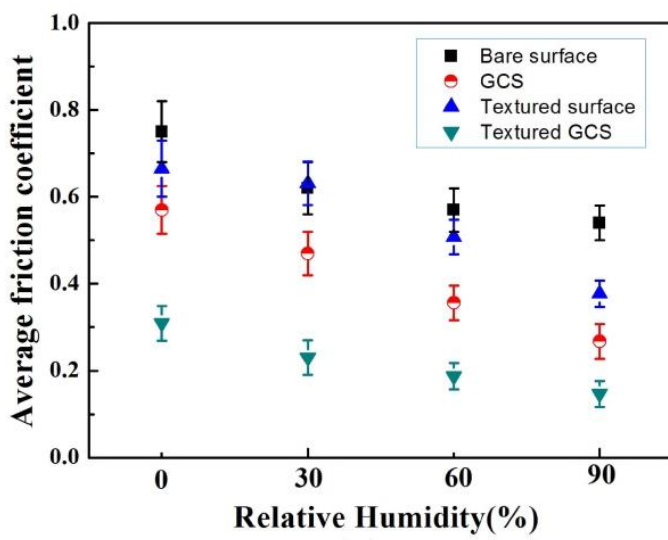

(a)

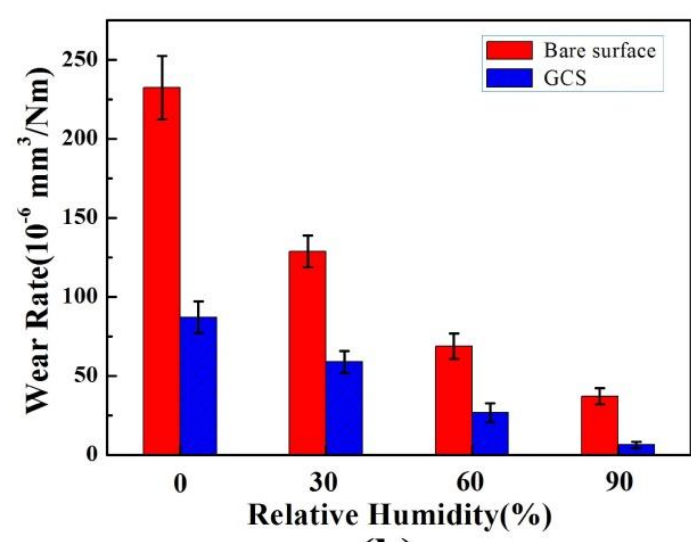

(b)

Fig. 5 Average COF of all test conditions (a) and wear rate of bare surface and GCS

(b)

\subsection{Wear surface analysis}

The SEM images of wear scar on the bare and textured GCS under different condition are shown in Fig. 6. From Fig. 6a and Fig. h, the wear scar under $\mathrm{N}_{2}$ condition is rough and underwent adhesive wear mechanism [25]. With the increase in $\mathrm{RH}$, the wear surface become smoother and exhibited an evident furrow trace with different depths and widths, indicating that the wear mechanism was abrasive wear. Meanwhile, the wear scar under high RH condition (Fig.6g and Fig. i) exhibits several irregular fine metal corrosion, and energy dispersive spectroscopy (EDS) analyses are carried out to estimate the oxygen element changes induced by humidity (each EDS location is marked by asterisk). As shown in Table 1, the oxygen content of the wear scar in $90 \% \mathrm{RH}$ is higher than that under $\mathrm{N}_{2}$ condition, revealing the existence of 
oxides. Hence, the wear mechanisms in high RH are abrasive wear and slight corrosion wear.
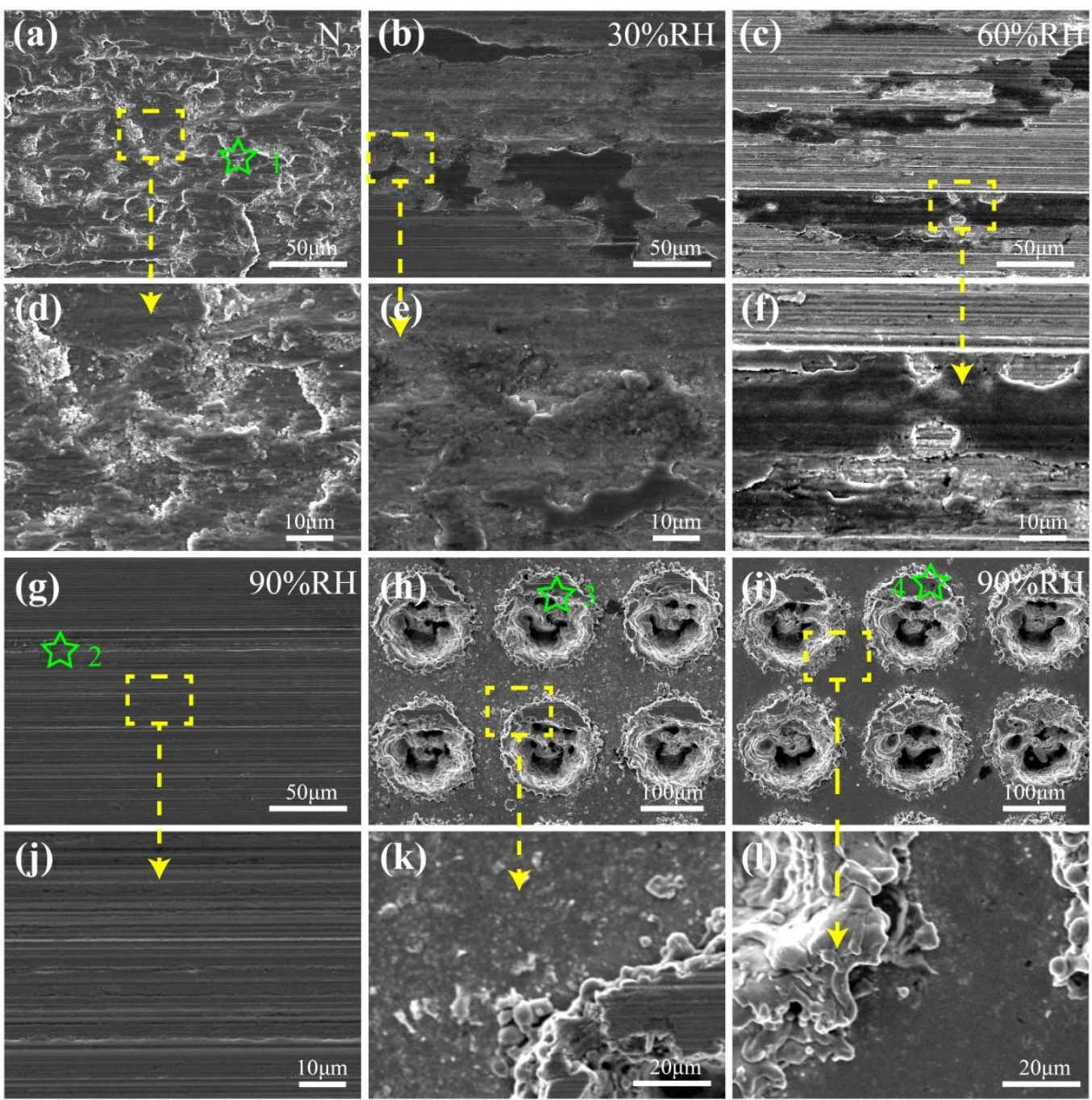

Fig. 6 SEM image of wear tracks on GCS and texture GCS under different conditions after $1200 \mathrm{~s}$ wear test.

Table 1 EDS analysis of wear tracks (wt.\%)

\begin{tabular}{rcccc}
\hline EDS area & $\mathrm{C}$ & $\mathrm{Fe}$ & $\mathrm{O}$ & $\mathrm{Mn}$ \\
\hline Spectrum 1 & 10.30 & 84.58 & 4.01 & 1.10 \\
Spectrum 2 & 6.69 & 83.75 & 8.58 & 0.98 \\
Spectrum 3 & 7.18 & 90.88 & 0.75 & 1.19 \\
Spectrum 4 & 7.41 & 85.09 & 6.35 & 1.15 \\
\hline
\end{tabular}


Fig. 7a shows the Raman spectra on the wear track of textured GCS after $1200 \mathrm{~s}$ wear test. As shown under $\mathrm{N}_{2}$ condition, graphene characteristics of $\mathrm{D}, \mathrm{G}$, and $2 \mathrm{D}$ peaks disappeared, and no evident peaks are observed in the Raman spectra, indicating that the structure of graphene was destroyed or graphene layer was removed out. This phenomenon was in accordance with the COF at $1200 \mathrm{~s}$, increasing to 0.49 and proving failure of lubrication. Similarly, the 2D peak do not appear in the Raman spectra of the wear track under $30 \%$ and $60 \%$ RH. However, the typical graphene Raman spectral characteristics exist in wear track under $90 \% \mathrm{RH}$, and the ratio of $2 \mathrm{D} / \mathrm{G}$ intensity increase compared to the original surface, indicating multilayer graphene transformation to a single layer. On the other hand, the D peak with large width and high ratio of D/G intensity indicates highly disordered and defect characteristics of the graphene layer. Hence, the Raman spectra analysis results of wear tracks are in accordance with the COF curve in Fig. 4d. The graphene layer was sheared during reciprocating sliding and was destroyed or removed out after $1200 \mathrm{~s}$ test under $\mathrm{N}_{2}$ condition because of serious adhesive wear.

To explore the durability of textured GCS in $90 \% \mathrm{RH}, 4000$ s wear test was carried out in Fig. 7b. The COF started to increase until 2600s, remained stable at 3100s, and was considerably longer than that of GCS. The Raman spectra analysis of original GCS and 1200s test GCS was discussed above. After test, the Raman spectra of the worn surface exhibit weak D, G, and 2D peak similar to the feature of the worn surface under $\mathrm{N}_{2}$ atmosphere after 1200s test, indicating that graphene was worn out. Interestingly, the graphene layer still existed in the textured sample after 1200s test under $90 \% \mathrm{RH}$ condition, and guess is proposed that the graphene layer on the surface was protected and repaired by water. 


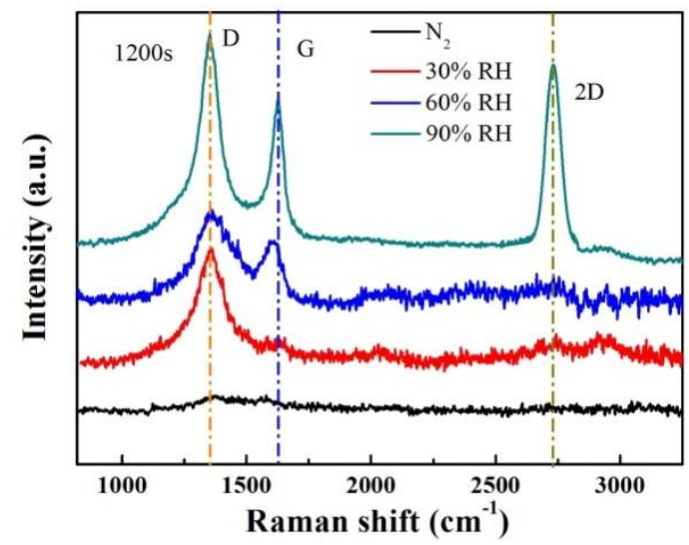

(a)

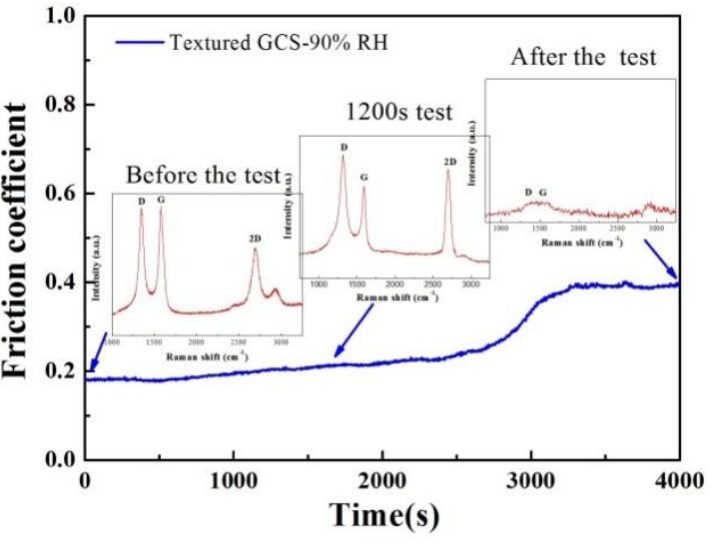

(b)

Fig. 7 Raman spectra on the wear track of textured GCS after $1200 \mathrm{~s}$ test under different RH (a) and textured GCS after 4000 s test under 90\% RH (b)

On the basis of the guess, we described the dynamical atomistic interactions to explain the mechanism during sliding in nitrogen and high RH atmosphere in Fig. 8. The initial graphene layer (Fig. 8b) was deposited on the steel surface (Fig. 8a), and then the graphene layer was ruptured under the severe contact pressure of about 0.45 GPa during friction (Fig. 8c), which means that the carbon atom undergoes bond breaking formed carbon dangling bonds $[6,7,26]$. When the fractured and defective graphene layer was exposed to the nitrogen atmosphere, we observe that only physical adsorption occurs on the graphene surface without repair and passivation on the ruptured section of graphene layer because the nitrogen molecule did not dissociate and react with the carbon dangling bonds in the broken area and fail to stabilize the broken area of graphene; thus, the ruptured edge of graphene cracked rapidly under high contact pressure and ultimately lost the lubrication ability (Fig. 8d). By contrast, when exposed to high $\mathrm{RH}$ condition, $\mathrm{H}$ and $\mathrm{OH}$ dissociated from water molecules according to first principles calculations [27], then appeared in the broken area of graphene layer (Fig. 8f) because $\mathrm{H}$ and $\mathrm{OH}$ could react with the carbon dangling bonds. Carbon dangling bonds of graphene was combined with $\mathrm{H}$ and $\mathrm{OH}$ transformed from $s p^{2}$ ( $\mathrm{C}=\mathrm{C}$ bonds) to $s p^{3}$ (C-C bonds) hybridization, and the schematic is presented in Fig. 8e, stabilizing and passivating the ruptured edge of 
graphene and protecting the graphene layer from further damage. The ratio of $\mathrm{D} / \mathrm{G}$ peak intensity under $90 \% \mathrm{RH}$ condition after 1200s testing increase contrast to original surface not also the reason of fracture and defective graphene layer during sliding but also due to the formation of $\mathrm{C}-\mathrm{H}$ and $\mathrm{C}-\mathrm{OH}$ bond in the graphene C-network. This phenomenon also occurs at other carbon systems, such as graphite, HOPG, graphite-like carbon film, and diamond-like carbon film [6, 7, 28-30], all of them shows a lower firction and wear in the humidity environments.

(a)

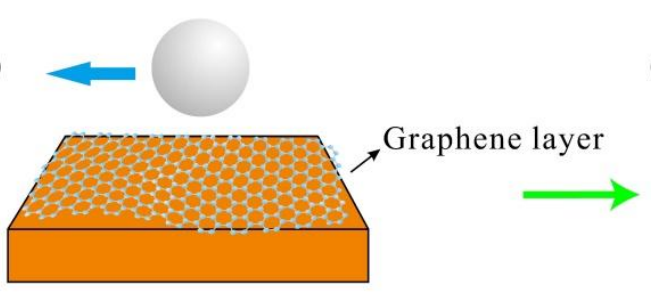

(e)
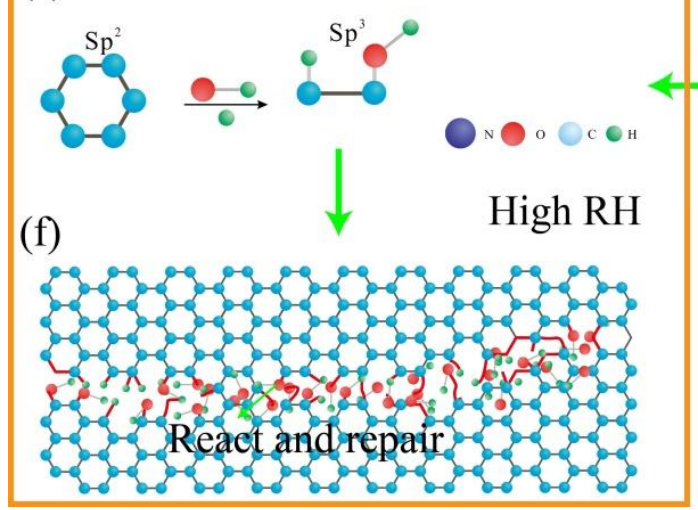

(b)

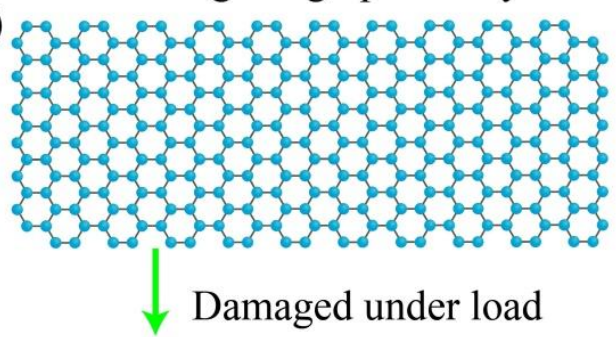

(c)

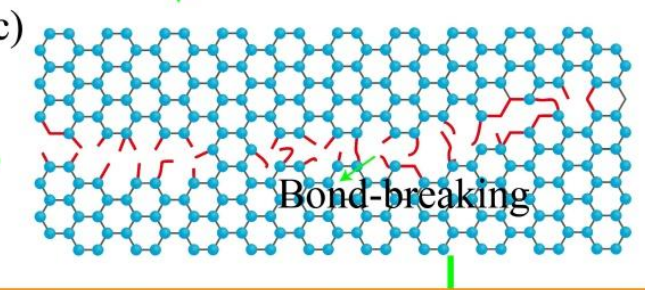

Original graphene layer

(d)

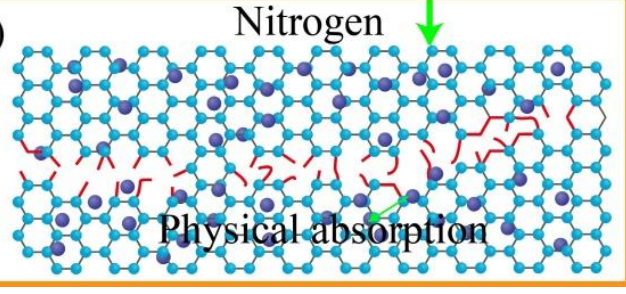

Fig. 8 Modification of graphene layer in nitrogen and high $\mathrm{RH}$ atmosphere

The X-ray photoelectron spectrum (XPS) of textured GCS after $1200 \mathrm{~s}$ wear test under $90 \% \mathrm{RH}$ is shown in Fig. 9. The XPS survey of the C 1 s, O 1s, and Fe 2p is clearly observed in Fig. 9a. The C 1s peaks (Fig. 9b) appearing at the bonding energies of 284.80, 286.20, and $288.80 \mathrm{eV}$ belonged to C-C/C-H, C-OH/C-O-C, and $\mathrm{O}-\mathrm{C}=\mathrm{O}$, respectively. The $\mathrm{O} 1 s$ peak is shown in Fig. 9c, with a broad asymmetric spectrum that indicates various oxygen bonding environments. The $\mathrm{O} 1 s$ peak is decomposed into three components, i.e., the binding energies of 530, 531.8, and 532.8 
$\mathrm{eV}$ attributed to $\mathrm{O}^{2-}, \mathrm{C}=\mathrm{O}$, and $\mathrm{C}-\mathrm{O}$, respectively [18, 31-32]. As shown in Fig.9d, the main peak of $\mathrm{Fe} 2 p$ observed at $710.97 \mathrm{eV}$ may be assigned to the $\mathrm{Fe} 2 p^{3 / 2}$ accompanied with a smaller peak of Fe metal at $707.10 \mathrm{eV}$, and the separation energy between the main $\mathrm{Fe} 2 p^{3 / 2}$ peak and its satellite peak is $9.0 \mathrm{eV}$, demonstrating the existence of $\mathrm{Fe}_{2} \mathrm{O}_{3}$ [33]. These results suggest that $\mathrm{H}$ and $\mathrm{OH}$ combined with the carbon atom in the graphene layer.

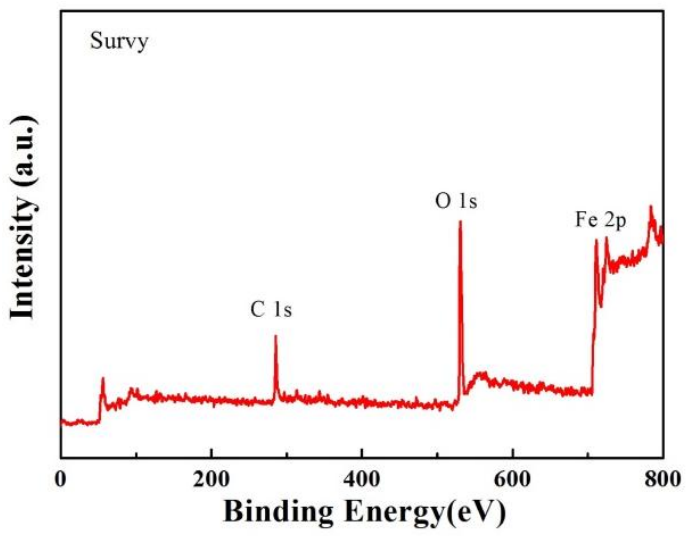

(a)

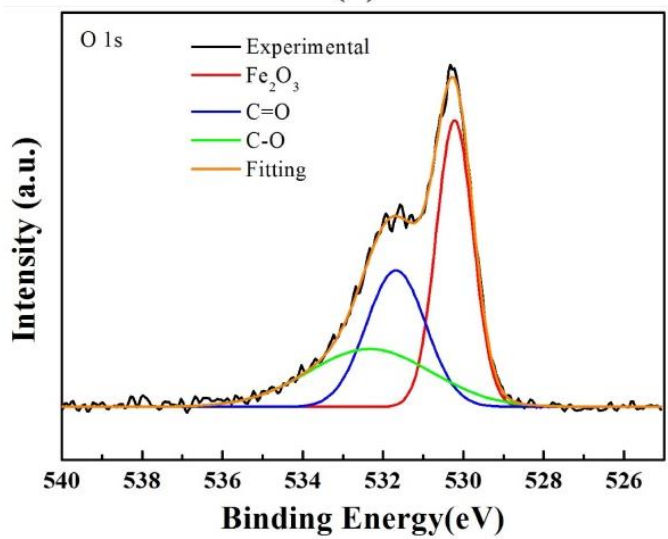

(c)

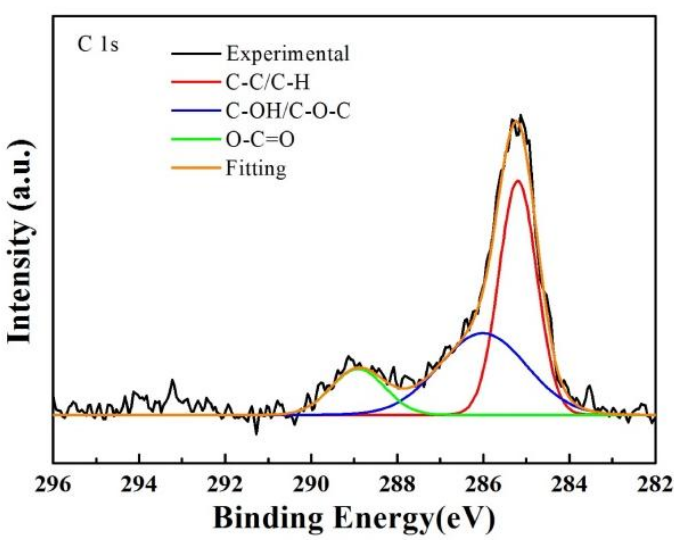

(b)

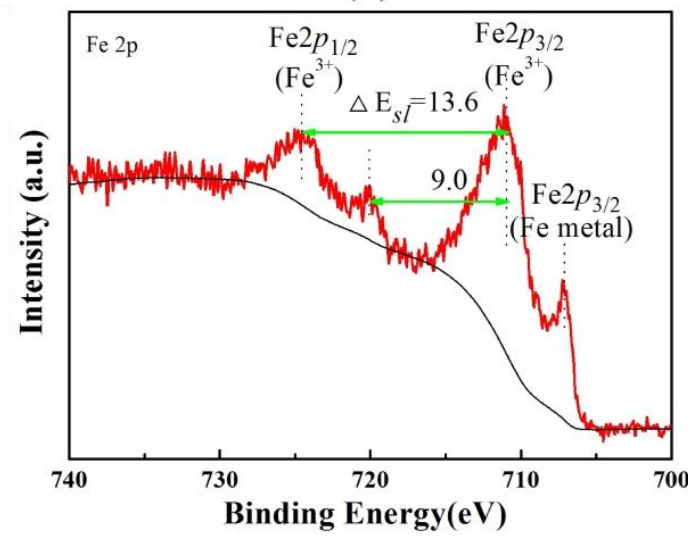

(d)

Fig. 9 XPS of textured worn scar after 1200 s under $90 \%$ RH

\subsection{Lubrication mechanism}

Based on the experimental results, the wear mechanism for longer duration of low COF under textured GCS was proposed. The graphene layer, which is irregularly distributed to the textured surface, is easily transferred and adheres to the counterpart 
ball surface in the process of friction because of low shear strength [34], and then the ball surface would form multi-layer graphene similar to the textured surface, which prevents direct steel to steel contact at the interface of the friction pairs. During the test, the graphene particle stored in the dimple is released, thus continuously offering a graphene layer to friction interface [35]. Meanwhile, the graphene layer on the ball and plate was ruptured under high contact stress, and the ruptured edge of the graphene layer reacted with $\mathrm{H}$ and $\mathrm{OH}$ dissociated from water molecules, slowing down the damage to graphene. On the textured surface, the graphene layer on the ball surface would periodically contact with water molecules because of the existence of dimples; hence, the graphene layer on the ball surface could be protected similarly to the plate surface. As a result, the textured GCS in $90 \%$ RH shows a minimum COF contrast to the other test condition.

\section{Conclusions}

In this paper, GCS was prepared and tribological tests were performed to explore the role of graphene layer to reduce the friction and wear under different $\mathrm{RH}(0 \%$ (dry nitrogen), 30\%, 60\% and 90\%).

(a) The Roman spectra and SEM image of GCS showed a few layer graphene was irregular deposited on the steel surface and tribological tests indicated that GCS can provide a low COF.

(b) The duration of low COF was obviously affected by relatively humidity, the duration of low COF was increased in $90 \% \mathrm{RH}$ contrasted to $\mathrm{N}_{2}$ atmosphere, owing to the water molecules passivated and repaired the graphene according to the Raman and XPS spectra.

(c) The textured GCS showed the longer duration of low COF than that of bare GCS, attributed to the dimple on the surface can protect graphene layer on the ball surface which transferred from plate sample.

(d) Graphene was an extremely good solid lubrication material due to low shear 
strength, could effectively reduce friction and wear especially applied to textured surface.

\section{Acknowledgments}

This study was supported by National Science Foundation of China (51375407 and U1530136), and Young Scientific Innovation Team of Science and Technology of Sichuan (No.2017TD0017), Thank Dr. Jin-fang Peng from SWJTU for XPS measurements.

\section{Reference}

[1] J.K. Lancaster, A review of the influence of environmental humidity and water on friction, lubrication and wear, Tribol. Int. 23 (1990) 371-389.

[2] R.H. Savage, Graphite lubrication, J. Appl. Phys. 19 (1948) 1 - 10.

[3] J.K. Lancaster, J.R. Pritchard, Influence of environment anpressure on the transition to dusting wear of graphite, J. Phys. D: Appl Phys. 14 (1981) 747 - 62.

[4] B.K. Yen, Influence of water vapor and oxygen on the tribologyof carbon materials with sp2 valence configuration, Wear 192 (1996) $208-215$.

[5] F. Guo, Y. Tian, Y. Liu, Ultralow friction between cemented carbide and graphite in water using three-step ring-on-ring friction test, Wear 352 (2016) 54-64.

[6] J.C. Rietsch, P. Brender, J. Dentzer, Evidence of water chemisorption during graphite friction under moist conditions, Carbon 55 (2013) 90-97.

[7] J. Xiao, L. Zhang, K. Zhou, Anisotropic friction behaviour of highly oriented pyrolytic graphite, Carbon 65 (2013) 53-62.

[8] W.J. Hyun, O.O. Park, B.D. Chin, Foldable Graphene Electronic Circuits Based on Paper Substrates, Adv. Mater. 25 (2013) 4729-4734.

[9] A.A. Balandin, S. Ghosh, W. Bao, Superior thermal conductivity of single-layer graphene, 
Nano. Lett. 8 (2008) 902-907.

[10] C. Xu, P.A. Brown, J. Lu, Electronic Properties of Halogen-Adsorbed Graphene, J. Phys. Chem. C 119 (2015) 17271-17277.

[11] M. Guo, D. Diao, L. Yang, Restructured graphene sheets embedded carbon film by oxygen plasma etching and its tribological properties, Appl. Surf. Sci. 357 (2015) 771-776.

[12] F. Wählisch, J. Hoth, C. Held, Friction and atomic-layer-scale wear of graphitic lubricants on SiC(0001) in dry sliding, Wear 200 (2013) 78-81.

[13] Y. Zhang, M. Dong, B. Gueye, Temperature effects on the friction characteristics of graphene, Appl. Phys. Lett. 107 (2015) 011601.

[14] D.I. Kim, S.M. Park, S.W. Hong, The periodicity in interfacial friction of graphene, Carbon 85 (2015) 328-334.

[15] D. Marchetto, C. Held, F. Hausen, F. Wählisch, M. Dienwiebel, Friction and wear on single-layer epitaxial graphene in multi-asperity contacts, Tribol. Lett. 48 (2012) 77-82.

[16] M.S. Won, O.V. Penkov, D.E. Kim, Durability and degradation mechanism of graphene coatings deposited on Cu substrates under dry contact sliding, Carbon 54 (2013) 472-481.

[17] K.S. Kim, H.J. Lee, C. Lee, Chemical vapor deposition-grown graphene: the thinnest solid lubricant, ACS Nano 5 (2011) 5107.

[18] S. Bhowmick, A. Banerji, A.T. Alpas, Role of humidity in reducing sliding friction of multilayered graphene, Carbon 87 (2015) 374-384. [19] D. Berman, A. Erdemir, A.V. Sumant, Reduced wear and friction enabled by graphene layers on sliding steel surfaces in dry nitrogen, Carbon 59 (2013) 167-175.

[20] D. Berman, A. Erdemir, A.V. Sumant, Few layer graphene to reduce wear and friction on sliding steel surfaces, Carbon 54 (2013) 454-459.

[21] D. Berman, S.A. Deshmukh, A. Erdemir, Extraordinary Macroscale Wear Resistance of One Atom Thick Graphene Layer, Adv. Funct. Mater. 24 (2014) 6640-6646.

[22] D. Berman, A. Erdemir, A.V. Sumant, Graphene: a new emerging lubricant, Mater. Today. 17 (2014) 31-42.

[23] X. Ling, J. Wu, L. Xie, Graphene-Thickness-Dependent Graphene-Enhanced Raman Scattering, J. Phys. Chem. C 117 (2013) 2369-2376. 
[24] D. Zheng, Z.B. Cai, M. X. Shen, Investigation of the tribology behaviour of the graphene nanosheets as oil additives on textured alloy cast iron surface, Appl. Surf. Sci. 387 (2016) 66-75.

[25] K. Zhang, J. Deng, J. Sun, Effect of micro/nano-scale textures on anti-adhesive wear properties of WC/Co-based TiAlN coated tools in AISI 316 austenitic stainless steel cutting, Appl. Surf. Sci. 355 (2015) 602-614.

[26] L. Pastewka, S. Moser, M. Moseler, Atomistic Insights into the Running-in, Lubrication, and Failure of Hydrogenated Diamond-Like Carbon Coatings, Tribol. Lett. 39 (2010) 49-61.

[27] Y. Qi, E. Konca, A.T. Alpas, Atmospheric effects on the adhesion and friction between non-hydrogenated diamond-like carbon (DLC) coating and aluminum - A first principles investigation, Surf. Sci. 600 (2006) 2955-2965.

[28] Y. Wang, J. Pu, J. Wang, Interlayer design for the graphite-like carbon film with high load-bearing capacity under sliding-friction condition in water, Appl. Surf. Sci. 311 (2014) 816-824.

[29] A.A. Gharam, M.J. Lukitsch, Y. Qi, Role of oxygen and humidity on the tribo-chemical behaviour of non-hydrogenated diamond-like carbon coatings, Wear 271 (2011) 2157-2163.

[30] H.I. Kim, J.R. Lince, O.L. Eryilmaz, Environmental Effects on the Friction of Hydrogenated DLC Films, Tribol. Lett. 21 (2006) 51-56.

[31] A. Banerji, A. Edrisy, V. Francis, Effect of bio-fuel (E85) addition on lubricated sliding wear mechanisms of a eutectic Al-Si alloy, Wear 311 (2014) 1-13.

[32] C.H. Choi, S.Y. Han, Y.W. Su, Z. Fang, L. Y. Lin, C. H. Chang, Fabrication of high-performance, low-temperature solution processed amorphous indium oxide thin-film transistors using a volatile nitrate precursor, J. Mater. Chem. C 3 (2015) 854-60.

[33] Y. Zhou, Z.B. Cai, J.F. Peng, Tribo-chemical behavior of eutectoid steel during rolling contact friction, Appl. Surf. Sci. 388 (2016) 40-48.

[34] C.P. Marega, L. Gomes, First-principles comparative study on the interlayer adhesion and shear strength of transition-metal dichalcogenides and graphene, Phys. rev. B 92 (2015) 73-174.

[35] Z. Shi, P. Shum, A. Wasy, Tribological performance of few layer graphene on textured M2 steel surfaces, Surf. Coat. Tech. 296 (2016) 164-170. 


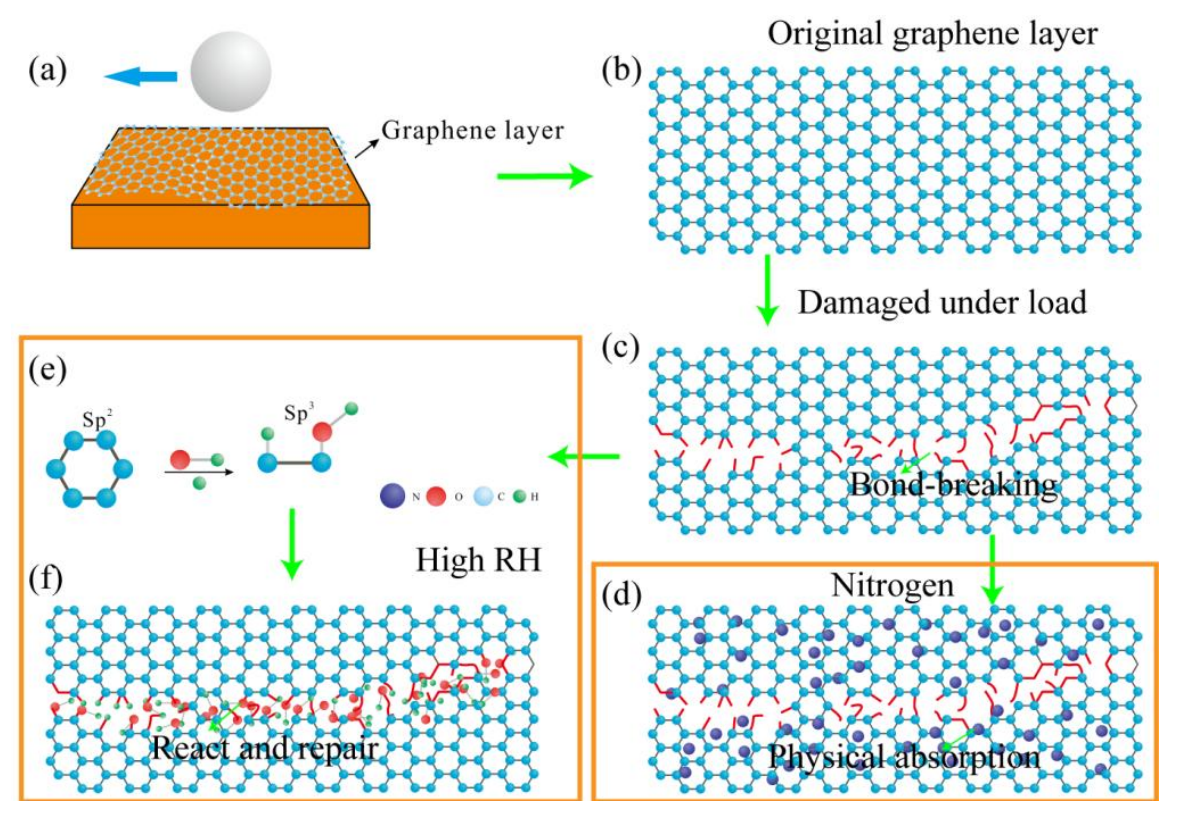

